\title{
Research of Quantum Entanglement Control and Decoherence
}

\author{
Zhaohua Wang ${ }^{\text {, }}$ Liqing Ren, Hanying Wang \\ College of Energy Engineering, Yulin University, Shaanxi 719000, China \\ awangzhaohua841102@163.com
}

Keywords: quantum entanglement control, de-coherence, Schrodinger's cat

\begin{abstract}
As the most peculiar and incredible feature of quantum mechanics, there is a great gap between quantum information and classical information, which is evident in the new field of quantum information. Based on this, this paper discusses quantum entanglement control and decoherence and hopes that it can bring some enlightenment for the related industry.
\end{abstract}

\section{Introduction}

For the quantum entanglement control of this paper, the information transmission and information processing which it provides for new physical resources should receive attention [1]. This is because such resources can provide strong support for the scientific development in this century [2]. The support can be implemented to practical application level from theory, it is the reason why this article focuses on quantum entanglement control and decoherence [3].

\section{Quantum Entanglement Control}

\subsection{Theoretical analysis}

When it comes to quantum entanglement control, imagination test "Schrodinger's cat", which is carried out by famous Austrian physicist Erwin Schrodinger in 1935, must be mentioned [4]. An intuitive visualization of the experiment "Schrodinger's cat" is shown in Figure 1, which is often seen as an important reason for the unsustainability of macroscopic quantum entanglement [5].

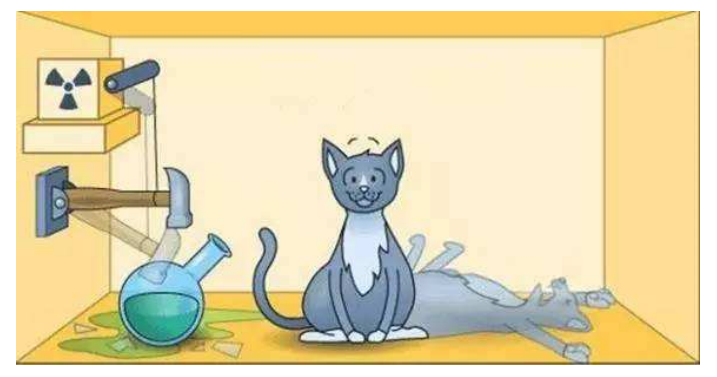

Fig.1 "Schrodinger's cat" experiment

The GHZ state belongs to a simple model of the "Schrodinger's cat" experiment, which is expressed as:

$$
|G H Z\rangle=\frac{1}{\sqrt{2}}\left(|0\rangle^{\otimes N}+|1\rangle^{\otimes N}\right)
$$

GHZ state possesses environmental phase effect of uncontrollable freedom degrees, such as hot bath. The in-depth analysis about GHZ is conducted and can draw the following conclusion, that is, refund entanglement rate of $\mathrm{N}$ quantum bit is $\mathrm{KN}$. The premise of this conclusion is that the disentanglement rate of the single quantum bit is $\mathrm{K}$.

The disentanglement rate analysis based on GHZ states does not fully describe the nature of quantum entanglement system. However, the disentanglement theory of multi-particle quantum 
system proposed by scholars in recent years can make up for this problem, and it is more mature and specific than the disentanglement rate. In the analysis which applies this theory to study GHZ states, we can confirm that the lifetime of GHZ state is directly affected by the number of ions $\mathrm{N}$. This effect is manifested as an increase of $\mathrm{N}$ and a shortened lifetime of the GHZ state. In a more in-depth study of GHZ in academia, some studies, which have studied the entanglement lifetimes of GHZ states, obtain the situation that entangled patches will greatly increase in coding entangled state after a specified time, and the entanglement life of quantum systems will also be significantly extended.

As a resource with high potential, the academic community has long been hoping to operate this new resource of quantum entanglement and ensure its long-term stability at the same time. Quantum entanglement control research in this paper is one of the representatives, this control is essentially an external "interference". After a comprehensive analysis of the study carried out by academia in recent years, it is not difficult to find that quantum dense coding control belongs to representatives in the field of research.

\subsection{Quantum entanglement control}

Suppose that A and B want to communicate with each other, A and B respectively have particles 1 and 2 at the two-level three-particle GHZ state, while C has particles 3. There into, states of 1, 2 and 3 can be described as:

$$
|\Psi\rangle_{123}=\frac{1}{\sqrt{2}}\left(|000\rangle_{123}+|111\rangle_{123}\right)
$$

Suppose the numbers 0-7 are encoded by quantum bit 1, 2 and 3, which can get the relationship table shown in Table 1.

Table 1 Correspondence of quantum states and the number

\begin{tabular}{clllllllll}
\hline Quantum states & $|000\rangle$ & $|001\rangle$ & $|010\rangle$ & $|011\rangle$ & $|100\rangle$ & $|101\rangle$ & $|110\rangle$ & $|111\rangle$ \\
\hline $\begin{array}{c}\text { Corresponding } \\
\text { numbers }\end{array}$ & 0 & 1 & 2 & 3 & 4 & 5 & 6 & 7 \\
\hline
\end{tabular}

Depending on the different numbers, A performs a transformation $\{I, X, Y, Z\}$ on its own quantum bit, which obtains the results shown in Table 2.

Table 2 Transformation and results of a on its quantum bit

\begin{tabular}{ccc}
\hline Numbers & Transformation & New state \\
\hline 0 & $I \otimes I \otimes I$ & $\frac{1}{\sqrt{2}}(|000\rangle+|111\rangle)$ \\
1 & $X \otimes I \otimes I$ & $\frac{1}{\sqrt{2}}(|100\rangle+|011\rangle)$ \\
2 & $X \otimes I \otimes I$ & $\frac{1}{\sqrt{2}}(|100\rangle+|011\rangle)$ \\
3 & $X \otimes I \otimes I$ & $\frac{1}{\sqrt{2}}(|100\rangle+|011\rangle)$ \\
4 & $Y \otimes I \otimes I$ & $\frac{1}{\sqrt{2}}(-|100\rangle+|011\rangle)$ \\
5 & $Z \otimes I \otimes I$ & $\frac{1}{\sqrt{2}}(|000\rangle-|111\rangle)$ \\
6 & $Z \otimes I \otimes I$ & $\frac{1}{\sqrt{2}}(|000\rangle-|111\rangle)$ \\
7 & $Z \otimes I \otimes I$ & $\frac{1}{\sqrt{2}}(|000\rangle-|111\rangle)$ \\
\hline
\end{tabular}

A further analysis can obtain changes and the results of $\mathrm{C}$ on its quantum bit as shown in Table 3 . 
Table 3 Changes and results of c on its quantum bit

\begin{tabular}{llll}
\hline Numbers & Primitive states & Operations & New state \\
\hline 0 & $\frac{1}{\sqrt{2}}(|000\rangle+|111\rangle)$ & $I \otimes I \otimes I$ & $\frac{1}{\sqrt{2}}(|000\rangle+|111\rangle)$ \\
1 & $\frac{1}{\sqrt{2}}(|100\rangle+|011\rangle)$ & $I \otimes I \otimes X$ & $\frac{1}{\sqrt{2}}(|101\rangle+|010\rangle)$ \\
2 & $\frac{1}{\sqrt{2}}(|100\rangle+|011\rangle)$ & $I \otimes I \otimes Y$ & $\frac{1}{\sqrt{2}}(-|101\rangle+|010\rangle)$ \\
3 & $\frac{1}{\sqrt{2}}(|100\rangle+|011\rangle)$ & $I \otimes I \otimes Z$ & $\frac{1}{\sqrt{2}}(|100\rangle-|011\rangle)$ \\
4 & $\frac{1}{\sqrt{2}}(-|100\rangle+|011\rangle)$ & $I \otimes I \otimes Z$ & $\frac{1}{\sqrt{2}}(-|100\rangle-|011\rangle)$ \\
5 & $\frac{1}{\sqrt{2}}(|000\rangle-|111\rangle)$ & $I \otimes I \otimes X$ & $\frac{1}{\sqrt{2}}(|001\rangle-|110\rangle)$ \\
6 & $\frac{1}{\sqrt{2}}(|000\rangle-|111\rangle)$ & $I \otimes I \otimes Y$ & $\frac{1}{\sqrt{2}}(-|001\rangle-|110\rangle)$ \\
7 & $\frac{1}{\sqrt{2}}(|000\rangle-|111\rangle)$ & $I \otimes I \otimes I$ & $\frac{1}{\sqrt{2}}(|000\rangle-|111\rangle)$ \\
\hline
\end{tabular}

A further analysis can divide numbers into two groups, namely the first group 0,5,6,7, and the second group 1,2,3,4. It can be drawn transformation and the results of $\mathrm{B}$ on the first quantum bit as Table 4 shown. Table 5 shows the correspondence between some numbers and quantum bits. Carrying out follow-up study according to thinking can achieve transmitting eight classic bits of information through the three quantum bit. Thus quantum dense coding control program is completed.

Table 4 Transforms and results of $b$ on the first quantum bit

\begin{tabular}{lllll}
\hline Numbers & Primitive states & Transformed states & $\begin{array}{l}\text { The second } \\
\text { quantum } \\
\text { bits }\end{array}$ & $\begin{array}{l}\text { The third } \\
\text { quantum bits }\end{array}$ \\
\hline 0 & $\frac{1}{\sqrt{2}}(|0\rangle+|1\rangle)$ & $\frac{1}{\sqrt{2}}(|0\rangle+|1\rangle+|0\rangle-|1\rangle)=|0\rangle$ & $|0\rangle$ & $|0\rangle$ \\
5 & $\frac{1}{\sqrt{2}}(|0\rangle-|1\rangle)$ & $\frac{1}{\sqrt{2}}(|0\rangle+|1\rangle-|0\rangle+|1\rangle)=|1\rangle$ & $|0\rangle$ & $|1\rangle$ \\
6 & $\frac{1}{\sqrt{2}}(-|0\rangle-|1\rangle)$ & $\frac{1}{\sqrt{2}}(-|0\rangle-|1\rangle-|0\rangle+|1\rangle)=-|0\rangle$ & $|0\rangle$ & $|1\rangle$ \\
7 & $\frac{1}{\sqrt{2}}(|0\rangle-|1\rangle)$ & $\frac{1}{\sqrt{2}}(|0\rangle+|1\rangle-|0\rangle+|1\rangle=|1\rangle$ & $|0\rangle$ & $|0\rangle$ \\
\hline
\end{tabular}


Table 5 correspondence of part numbers and quantum bit

\begin{tabular}{cccc}
\hline Numbers & $\begin{array}{l}\text { The first quantum } \\
\text { bit }\end{array}$ & $\begin{array}{l}\text { The second } \\
\text { quantum bits }\end{array}$ & $\begin{array}{l}\text { The third quantum } \\
\text { bit }\end{array}$ \\
\hline 0 & $|0\rangle$ & $|0\rangle$ & $|0\rangle$ \\
5 & $|1\rangle$ & $|0\rangle$ & $|1\rangle$ \\
6 & $-|0\rangle$ & $|0\rangle$ & $|1\rangle$ \\
7 & $|1\rangle$ & $|0\rangle$ & $|0\rangle$ \\
\hline
\end{tabular}

Figure 2 is another quantum entanglement control's scheme diagram. The figure shows that two quantum bits are associated with a common environment. The final entanglement situation can bring some inspiration for this study. Figure 3 shows the evolution of entanglement degree with time scale under different initial parameters obtained in practical application of this control scheme. Combining with this figure, the value of this control scheme can be well understood.

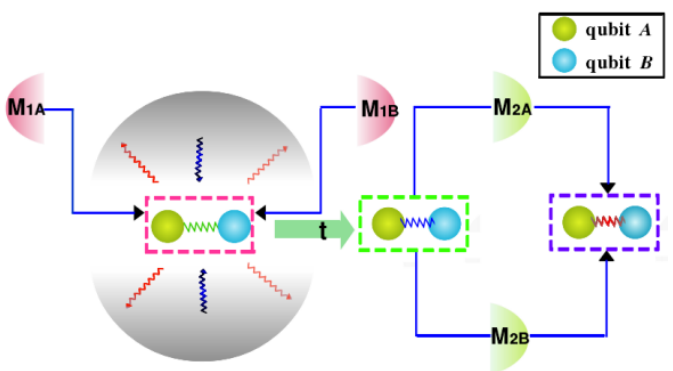

Fig.2 Another quantum entanglement control scheme diagram

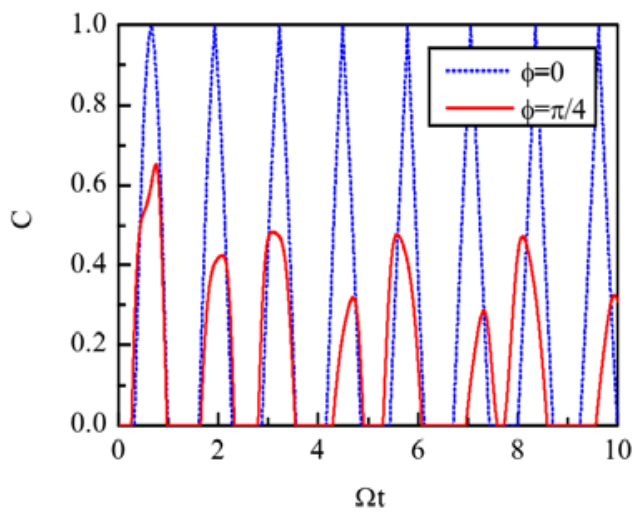

Fig.3 the evolution of entanglement degree with time scale under different initial parameters obtained in practical application

\section{Decoherence Theory}

After the analysis of the interaction between the quantum computer and the environment, it is not difficult to find two kinds of destruction process in this process, namely quantum decoherence and quantum dissipation. One of the quantum decoherence will lead to changes in the quantum computing encoding state, that is, quantum coherent superposition state is transformed into a classical state, and quantum dissipation will lead to computer energy loss. According to the research carried out by academia in recent years, it is clear that the quantum decoherence scale is smaller than the quantum dissipative practice scale, and the computational problems caused by it are just the key points in academic research in recent years.

If the quantum computer's environmental thermal bath and quantum entanglement resources lead to the decay of the diagonal elements of the density matrix, which indicates that the energy of the 
computer has dissipated. However, the re-entanglement of same tense and the environmental states is due to the evolution of the composite system, and the non-diagonal terms of the density matrix tend to be zero. However, if both the environmental state and the initial state of the computer state are in a pure state, that is

$$
\left|\varphi_{0}\right\rangle=\left[\sum_{i} c_{i}(0)|i\rangle\right] \otimes\left|a_{0}\right\rangle
$$

After an analysis around it, we can find that $\left\{\left|a_{i}(t)\right\rangle\right\}$ is gradually distinguishable and eventually becomes mutually orthogonal. The appearance of this situation and the function of the measuring instrument on the quantum system, the non-diagonal elements of the density matrix will gradually become zero. Quantum bit type, the role of the environment and the emergence of this situation are more closely linked. In order to really solve the decoherence problem, mixed-density composite matrix support is indispensable.

\section{Conclusions}

In summary, the research on quantum entanglement control and decoherence possesses high academic and practical value. On this basis, the two quantum entanglement control schemes involved in this paper prove the practical value of the research. Therefore, in the related field of theoretical research and practical exploration, the content of this article can play a certain reference role.

\section{Acknowledgements}

This work was supported by Shaanxi science and technology research and development plan project:2016GY-196; Shaanxi science and technology research and development plan project: 2014K05-11.

\section{References}

[1] Hou S C, Huang X L, Yi X X. Suppressing decoherence and improving entanglement by quantum-jump-based feedback control in two-level systems[J]. Physical Review A, 2010, 82(1):131-133.

[2] Pourkarimi M R, Rahnama M, Rooholamini H. Decoherence Effect on Quantum Correlation and Entanglement in a Two-qubit Spin Chain[J]. International Journal of Theoretical Physics, 2015, 54(4):1085-1097.

[3] Zhang J S, Chen A X. Enhancement of genuine multipartite entanglement and purity of three qubits under decoherence via bang-bang pulses with finite period[J]. Quantum Information Processing, 2016, 15(8):1-15.

[4] Tan Q Y, Wang L, Li J X, et al. Amplitude-Damping Decoherence Suppression of Two-Qubit Entangled States by Weak Measurements[J]. International Journal of Theoretical Physics, 2013, 52(2):612-619.

[5] Rahimi, Robabeh, SaiToh, Akira, Nakahara, Mikio. Bang-Bang Control of Entanglement in Spin-Bus-Boson Model[J]. Journal of the Physical Society of Japan, 2010, 76(11):2491-2511. 